\title{
Genome-wide association mapping of quantitative resistance to sudden death syndrome in soybean
}

\author{
Zixiang Wen', Ruijuan Tan', Jiazheng Yuan', Carmille Bales', Wenyan Du', Shichen Zhang ${ }^{1}$, Martin I Chilvers ${ }^{1}$, \\ Cathy Schmidt ${ }^{2}$, Qijian Song ${ }^{3}$, Perry B Cregan ${ }^{3}$ and Dechun Wang ${ }^{1 *}$
}

\begin{abstract}
Background: Sudden death syndrome (SDS) is a serious threat to soybean production that can be managed with host plant resistance. To dissect the genetic architecture of quantitative resistance to the disease in soybean, two independent association panels of elite soybean cultivars, consisting of 392 and 300 unique accessions, respectively, were evaluated for SDS resistance in multiple environments and years. The two association panels were genotyped with 52,041 and 5,361 single nucleotide polymorphisms (SNPs), respectively. Genome-wide association mapping was carried out using a mixed linear model that accounted for population structure and cryptic relatedness.
\end{abstract}

Result: A total of 20 loci underlying SDS resistance were identified in the two independent studies, including 7 loci localized in previously mapped QTL intervals and 13 novel loci. One strong peak of association on chromosome 18, associated with all disease assessment criteria across the two panels, spanned a physical region of $1.2 \mathrm{Mb}$ around a previously cloned SDS resistance gene (GmRLK18-1) in locus Rfs2. An additional variant independently associated with SDS resistance was also found in this genomic region. Other peaks were within, or close to, sequences annotated as homologous to genes previously shown to be involved in plant disease resistance. The identified loci explained an average of $54.5 \%$ of the phenotypic variance measured by different disease assessment criteria.

Conclusions: This study identified multiple novel loci and refined the map locations of known loci related to SDS resistance. These insights into the genetic basis of SDS resistance can now be used to further enhance durable resistance to SDS in soybean. Additionally, the associations identified here provide a basis for further efforts to pinpoint causal variants and to clarify how the implicated genes affect SDS resistance in soybean.

Keywords: Genome wide association mapping, Glycine max, Fusarium virguliforme, SNPs, Quantitative trait loci mapping

\section{Background}

Sudden death syndrome (SDS) of soybean [Glycine $\max ($ L.) Merr.], caused by the soil-borne fungal pathogen Fusarium virguliforme [1], is a considerable threat to soybean production [2]. The fungus infects soybean root systems and produces toxins that are translocated to the leaves, resulting in premature defoliation and pod abortion $[3,4]$. In recent years, SDS ranked among the top five most damaging diseases of soybean in the United States [5]. In the Midwestern soybean producing area of the U.S., it is estimated that SDS has resulted in average losses valued at $\$ 190$ million a year [6].

\footnotetext{
* Correspondence: wangdech@msu.edu

'Department of Plant, Soil and Microbial Sciences, Michigan State University, 1066 Bogue St., Rm. A384-E, East Lansing, Ml 48824, USA

Full list of author information is available at the end of the article
}

Host plant resistance is believed to be the most effective control measure for SDS [7]. Since no soybean genotypes confer complete immunity to this disease, soybean breeders still rely on quantitative resistance to SDS $[7,8]$. The wide range of variation of susceptibility to both leaf scorch and root rot also provides a great opportunity to improve SDS resistance through genetic manipulation [9]. However, most of what we know about the genetic architecture of SDS resistance is based on traditional quantitative trait locus (QTL) linkage mapping using biparental populations. Fourteen QTLs dispersed throughout the genome, underlying resistance to root infection, leaf scorch or both, have been confirmed in several biparental populations [10]. However, the large confidence intervals for those QTLs impair the precise identification of causative genes. To date, only one resistance gene (GmRLK18-1) has been tagged and cloned [11]. This 
gene is at $1.071 \mathrm{kbp}$ on chromosome 18 , within the major resistance QTL (Rfs2). Association mapping, which exploits historical recombination events at the population level, has become a powerful alternative to linkage mapping in the dissection of complex trait variation at the sequence level [12]. A more specific strategy, genome-wide association (GWA) mapping, is a powerful complementary strategy for classical bi-parental linkage mapping to dissect complex traits and has been used with success in Arabidopsis [13], rice [14] and maize [15,16]. The use of association mapping in soybean was therefore desirable to improve the mapping of important traits in soybean. So far, only a few association mapping studies, with limited numbers of markers, have been reported for dissecting agronomic traits in soybean $[17,18]$. To the best of our knowledge, GWA mapping has not yet been employed to study any traits related to soybean disease resistance. Recently, the availability of a soybean reference genome sequence and the development of high throughput SNP assays has enabled GWA mapping in soybean $[19,20]$. A previous study reported that approximately $1 \%$ of the 6,037 Plant Introductions (PIs) from the USDA Soybean Germplasm Collection were partially resistant to SDS [21]. Therefore, conducting an association study in assembled PI collections might not be feasible. Furthermore, previous research indicated that most of the PIs showing resistance to soybean cyst nematode ( $\mathrm{SCN}$ ) were also partially resistant to SDS [22]. Therefore, association mapping with released elite cultivars is more likely to identify superior resistance alleles that have been captured and accumulated by SCN or SDS breeding practices.

The goal of this study was to investigate the genetic architecture of soybean SDS resistance in released elite soybean cultivars. Here we present the first experimental results of GWA mapping for SDS, across two independent panels of elite soybean cultivars, using a high-density customized oligonucleotide genotyping array. We detected 20 QTLs including known candidate genes (or QTLs) as well as new candidate loci in the soybean genome. The identification of these loci will increase our understanding of mechanisms underlying SDS resistance, and provide valuable markers for breeding soybean lines with SDS resistance.

\section{Methods}

\section{Sampling and genotyping}

Two independent experiments were conducted in this study. Experiment 1 was done with a mapping population of 392 diverse soybean cultivars (association panel P1), consisting of 251 varieties released between 2010 and 2012 and 141 advanced breeding lines from Michigan State University. Experiment 2 used a set of 300 diverse G. max advanced breeding lines (association panel P2) developed by public breeders. The germplasm was chosen to represent a range of materials developed for the U.S. North Central soybean production area. Further information about the P1 and P2 panels is given in Additional file 1.

Soybean genomic DNA was extracted from young leaf tissue following the previously described method [23]. All the accessions in panel P1 were genotyped using the Illumina SoySNP50k iSelect BeadChip (Illumina, San Diego, Calif. USA) which consists of 52,041 SNPs [20]. All the accessions in panel P2 were genotyped using the Illumina SoySNP6k iSelect BeadChip (Illumina, San Diego, Calif. USA), which consists of 5,361 SNPs [24]. The chromosomal distributions of the SNPs of SoySNP50K and SoySNP6k BeadChip are shown in Additional file 2. Genotypes were called using the program GenomeStudio (Illumina, San Diego, Calif. USA). The SNP data were coded according to the standard codes for nucleotides derived from the International Union of Pure and Applied Chemistry (IUPAC). The quality of each SNP was checked manually as previously reported [25]. SNPs without physical position information and with low quality (call rate $<80 \%$ and or minor allele frequency $<0.05$ ) across all samples were removed from the dataset.

\section{Field resistance evaluation}

The association panel P1 was evaluated for SDS resistance in a naturally infested SDS disease nursery at Decatur, Michigan during the growing season (May-October) in 2011 and 2012, where consistent, natural and heavy SDS disease symptoms was observed on susceptible checks. Four replications per year were grown in a lattice design with four-row plots 6 meters long. The association panel P2 was divided into four groups based on the maturity group I to IV, and were evaluated for SDS resistance during the summers of 2011 and 2012 in 14 locations including Michigan (Decatur), Iowa (Kanawha and Ames), Minnesota (Waseca and Rosemount), Illinois (Manito, Streator, Fairbury, Beardstown, Urbana, Shawnee town and Valmeyer), Missouri (Sikeston) and Ontario, Canada (Harrow). Four replications per year were grown in a lattice design. SDS was evaluated by scoring disease incidence (DI) and disease severity (DS) at the R6 growth stage, the stage at which pods contain full-size green beans at one of the four uppermost nodes with a completely unrolled leaf [26]. SDS leaf scorch DI was rated from 0\% (no disease) to $100 \%$ (all plants symptomatic), and DS was measured on a scale from 1 to 9 as described in Additional file 3 (after Bond, J. unpublished). The SDS disease index (DX, 0-100) was calculated as DI $\times$ DS/9. In panel P1, mean values of DI, DS and DX across replicates and years were used in association analysis throughout the study. The trait distribution for DX and DI was slightly skewed towards susceptible, thus a square root transformation was used to normalize the trait distribution prior to further analysis. The association panel 
P2 was phenotyped in multiple environments; best linear unbiased predictors (BLUPs) were used for the overall association analysis in panel P2. The BLUPs for each line were calculated with the $\mathrm{R}$ package, lme4, using the equation $Y_{i j k}=$ Line $_{k}+$ Environment $_{i}+$ Replicate (Environment $)_{i j}+(\text { Line } \times \text { Environment })_{i k}+\varepsilon_{i j k}$, where $Y_{i j k}$ is the observed phenotype for the $k^{\text {th }}$ line in the $j^{\text {th }}$ replicate of the $i^{\text {th }}$ environment; Line $_{\mathrm{k}}$ is the random effect of the $k^{\text {th }}$ line; Environment $t_{i}$ is the random effect of the $i^{\text {th }}$ environment; Replicate (Environment) $)_{i j}$ is the random effect of the $j^{\text {th }}$ replicate in the $i^{\text {th }}$ environment; (Environment $\times$ Line $)_{i k}$ is the random interaction effect of the $i^{\text {th }}$ environment and the $k^{\text {th }}$ line, and $\varepsilon_{i j k}$ is the error term. Analysis of variance (ANOVA) for the phenotypic data was performed with the $\mathrm{R}$ package, $\operatorname{lm}$ (stats) and anova.Im(stats). The heritability estimates were calculated using variance components obtained by ANOVA [27].

\section{Population genetic analysis}

Principal components analysis and neighbor-joining (NJ) trees were applied to infer population stratification. A pairwise distance matrix derived from the Nei's genetic distance for all polymorphic SNPs was calculated to construct Neighbor-joining trees under PowerMarker version 3.25 [28]. Principal component analysis (PCA) was done using EIGENSTRAT [29] based on 5,578 SNPs and 2,587 SNPs with minor allele frequency (MAF) $>20 \%$ and physical distance $>60 \mathrm{~kb}$ for panels P1 and P2, respectively. Kinship matrices were calculated using TASSEL 4.0 [30] to determine relatedness among individuals based on the same sets of SNPs for the two panels [see Additional file 4]. Linkage disequilibrium parameter $\left(r^{2}\right)$ for estimating the degree of LD between pair-wise SNPs (30,345 SNPs for panel P1 and 4,297 SNPs for panel P2 with MAF $\geq 5 \%$ ) was calculated using the software TASSEL 4.0 [30] with 1,000 permutations. The LD decay rate was measured as the chromosomal distance at which the average pairwise correlation coefficient $\left(r^{2}\right)$ dropped to half its maximum value.

\section{Genome-wide association analysis}

Two different models were used to test associations between the SNPs (MAF >5\%) and disease assessment criteria. The first model was a simple model where a general linear model (GLM), containing only the SNP tested as a fixed effect, was used to test the association between the SNP and the disease assessment criteria. The second model is a mixed linear model (MLM) where, in addition to the SNP being tested, PCA matrix and relative kinship matrix were included as fixed and random effects, respectively. The GLM and MLM can be expressed as $y=X \alpha+e$ and $y=X \alpha+P \beta+K \mu+e$, respectively, where $y$ is the vector of phenotypic observations, $\alpha$ is the vector of SNP effects, $\beta$ is the vector of population structure effects, $\mu$ is the vector of kinship background effects, $e$ is the vector of residual effects, $P$ is the PCA matrix relating $y$ to $\beta, X$ and $K$ are incidence matrices of $1 \mathrm{~s}$ and $0 \mathrm{~s}$ relating $y$ to $\alpha$ and $\mu$, respectively [31]. Top six principal components were used to build up the $P$ matrix for population structure correction in the two panels. Analyses were performed by the software TASSEL 4.0 which implemented the EMMA and P3D algorithms to reduce computing time [32]. False discovery rate $(F D R) \leq 0.05$ was used to identify significant associations. In order to conduct conditional analyses to test for residual adjacent associations after accounting for a key SNP within the same chromosome, the key SNP was transformed to a numeric value and then added into the MLM as a covariate. A $P$-value threshold of $10^{-4}$, corresponding to an adjustment for 500 independent tests across the region examined, was used to declare statistical significance at secondary signals.

\section{Results and discussion}

\section{Genetic diversity and phenotypic variation}

Two independent association panels (P1 and P2) were genotyped using Illumina BeadChip containing 52,041 and 5,361 SNPs, respectively. SNPs with MAF of $<0.05$ and call rate $<80 \%$ were excluded from further analyses to avoid problems of spurious LD. Final sets of 30,345 and 4,297 high-performing SNPs were used for all analyses. Among these SNPs, samples had an average call rate of $>96.5 \%$ and between technical replicates yielded $>99 \%$ pairwise concordances. From these SNPs, we observed an average nucleotide diversity (polymorphism information content or PIC) of 0.281 and 0.284 in panels P1 and P2, respectively. Compared with a previous study [33], these estimates showed that the overall genetic variation of the elite cultivars we studied represents about $80 \%$ diversity of soybean landraces. Less than $1.6 \%$ of heterozygous genotypes were observed in both panels, which is consistent with the highly inbred nature of cultivated soybean (Table 1). An examination of allele frequency distributions at polymorphic SNPs showed that both panels contained a large number of SNPs with a minor allele frequency (MAF) of $<0.1$ [see Additional file 5], reflecting the broad genetic diversity in the two association panels.

In both association panels, we observed abundant phenotypic variation in SDS resistance measured by disease incidence (DI), disease severity (DS) and calculated disease index (DX, see Additional file 6). The mean DX distribution ranged from 0 to 96.3 in panel P1 and 0 to 82.0 in panel P2. The broad-sense heritability of DX was higher within two environments (two years in one location) in panel P1 (83\%) than that across multiple environments (two years across 14 locations) in panel P2 (average 65\%) [see Additional file 6]. 
Table 1 Characteristics of SNPs tested in two association panels

\begin{tabular}{lcccccc}
\hline & Total SNPs & Polymorphic SNPs & MAF $^{\mathbf{b}}>\mathbf{0 . 0 5}$ & Density (kb/SNP) & PIC $^{\mathbf{c}}$ & Heterozygosity rate \\
\hline P1 $\left(392^{\mathbf{a}}\right)$ & 52,041 & 39,554 & 30,345 & 35 & 0.281 & $1.4 \%$ \\
P2 $(300)$ & 5,361 & 5,132 & 4,297 & 241 & 0.284 & $1.6 \%$ \\
\hline
\end{tabular}

${ }^{a}$ No. of accessions; ${ }^{b} \mathrm{MAF}$, minor allele frequency; ${ }^{\mathrm{C}} \mathrm{PIC}$, polymorphism information content.

\section{Patterns of linkage disequilibrium}

To characterize the mapping resolution for genome scans and GWA mapping, we quantified the average extent of genome-wide LD decay distance in panel P1 and P2. These estimates were approximately $270 \mathrm{~kb}$ and $460 \mathrm{~kb}$, respectively, where the $r^{2}$ drops to half its maximum value ( 0.24 and 0.19 , respectively). Given that our average inter-marker distance (density) is $35 \mathrm{~kb}$ and $241 \mathrm{~kb}$ for the panels P1 and the P2 respectively, we expect to have reasonable power to identify common large effect variants associated with SDS resistance in both association panels. Overall LD decay distance in panel P1 was smaller than that in the panel P2 (Figure 1). LD decay distance in panel P1 was also smaller than previously published values in soybean [33,34]. This difference may be attributed to smaller sample size and lower genome coverage of markers in P2 and previous studies. Since panel P1 had larger sample size and was genotyped with more SNP markers than panel $\mathrm{P} 2$, estimation of $\mathrm{LD}$ in panel $\mathrm{P} 1$ is more reliable. Linkage disequilibrium decay distance varies over different chromosomes, with $410 \mathrm{~kb}$ in chromosome 19, 100-200 kb in chromosomes 3, 4, 9, 10, 11 and 16, and $200-300 \mathrm{~kb}$ in the remaining 13 chromosomes in panel P1. These LD decay estimates are slightly higher than that in rice $(75-150 \mathrm{~kb})$ [14], but much greater than in maize $(1.5-10 \mathrm{~kb})$ [35]. This result is consistent with earlier estimate that LD extends to a much longer distance in self-pollinated species than in cross-pollinated species [12].

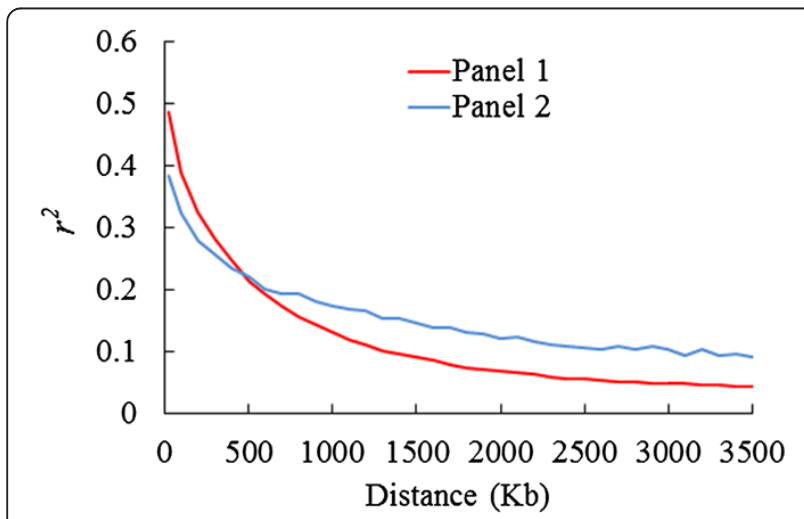

Figure 1 Genome-wide average LD decay estimated in association panels P1 and P2. Decay of LD (measured as genotypic $r^{2}$ ) as a function of distance between SNPs.

\section{Population structure}

According to the NJ tree analysis as well as PCA, association panel P1 had 4 genetic subgroups (Figure $2 \mathrm{a}$ and b), whereas panel P2 had 6 subgroups (Figure 2c and d). It has previously been suggested that the photoperiod response between different maturity groups may be the primary factor driving differentiation of cultivated soybean [36]. A Chi-square test was used to test whether the SNP-data-based clustering (NJ tree) is associated with maturity-group-based grouping in panel P1 and P2. The results showed very significant association $(P<0.0001)$ between the two grouping factors. Thus the photoperiod response might have driven genetic differentiation among the tested accessions in both panels [see Additional file 7]. The measure of population differentiation, $F_{\mathrm{ST}}$, was estimated at 0.168 among the four subgroups of panel P1, suggesting a moderate level of differentiation within panel P1 [see Additional file 8]. The population differentiation of 6 subgroups within panel $\mathrm{P} 2$ was slightly less $\left(F_{\mathrm{ST}}=0.135\right)$ but still similar to that between different soybean landraces $\left(F_{\mathrm{ST}}=0.130\right)[33]$.

\section{GWA mapping for SDS resistance}

Using the GWA strategy to dissect genetic architecture of SDS resistance in the two soybean association panels (P1 and P2), we successfully identified both known associations (candidate genes or QTLs previously reported in soybean), as well as new candidate loci in the soybean genome. The results of significant SNPs discovered in both association panels are summarized in Additional file 9, 10, 11, 12, 13, 14, and Figure 3. As shown in the quantile-quantile (QQ) plots (Figure $3 \mathrm{~b}$ and $\mathrm{f}$, Additional file $13 \mathrm{~b}$ and $\mathrm{f}$, and Additional file $14 \mathrm{~b}$ and $\mathrm{f}$ ), the distribution of observed $\log 10 P$-values from the simple model, which did not include population structure (Q) and familial relatedness $(\mathrm{K})$, departed from the expected distribution under a model of no association with significant inflation of nominal P-values. While the MLM method, which includes $\mathrm{Q}$ and $\mathrm{K}$, allowed us to reduce the excess low P-values for DS, DI and DX (Figure 3d and h, Additional file 13d and h, and Additional file 14d and $\mathrm{h}$ ). In both association panels, lower inflation of nominal $P$-values was consistently observed when the MLM method was used than when the simple model was used. Therefore, only the results from the analysis with the MLM model are presented below. 

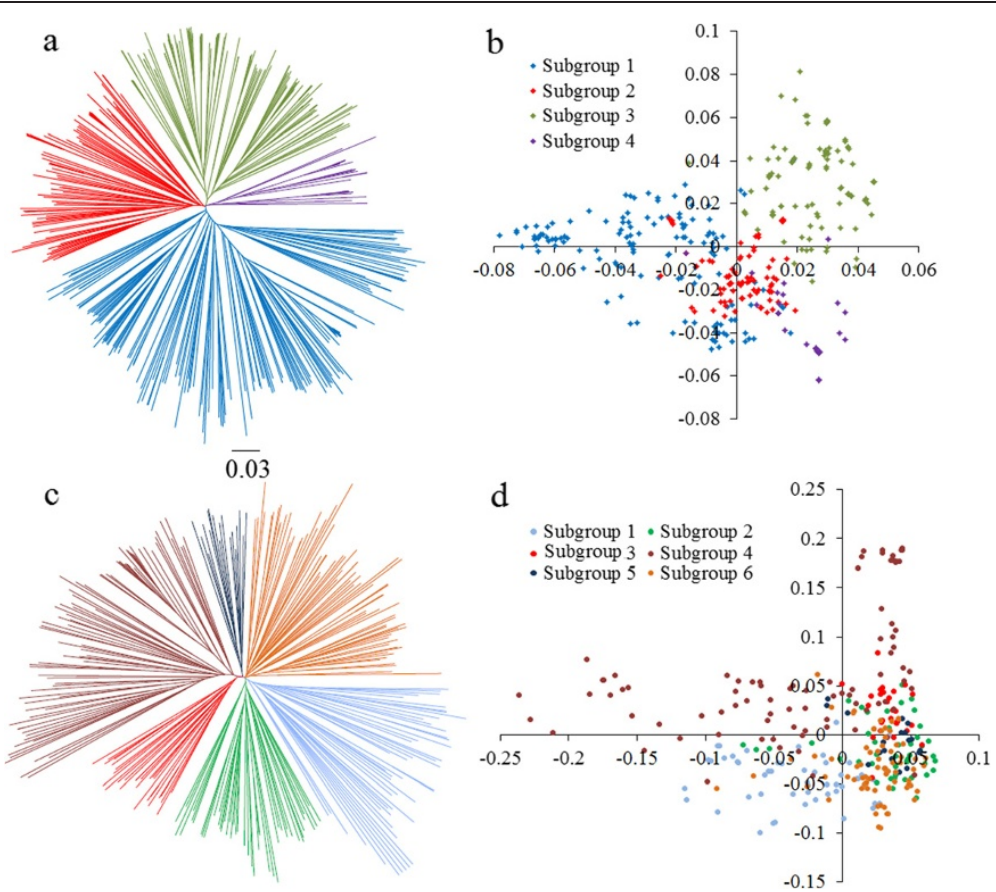

Figure 2 Population structures of soybean cultivars in association panels P1 and P2. (a) Neighbor-joining tree of 392 accessions in panel P1. The four subgroups identified from the tree are color-coded in $\mathbf{a}$ and $\mathbf{b}$. (b) PCA plots of the first two components of 392 accessions in panel P1. (c) Neighbor-joining tree of 300 accessions in panel P2. The six subgroups identified from the tree are color-coded in c and $\mathbf{d}$. (b) PCA plots of the first two components of 300 accessions in panel P2.

Of the 52,041 SNPs evaluated in association panel P1, 30, 48 and 56 SNPs were significant, with FDR $\leq 0.05$ for DS, DI and DX, respectively (Additional file 9, 10, 11, Figure $3 \mathrm{a}$ and c, Additional file 13a and c, and Additional file 14a and c). From the 5,361 SNPs evaluated in association panel P2, 6, 8 and 9 SNPs were significant, with FDR $\leq 0.05$ for DS, DI and DX, respectively (Figure 3e and g, Additional file 12, Additional file 13e and $\mathrm{g}$ and Additional file 14e and g). To select major QTLs among all the significant SNPs, these SNPs were clumped by using LD block as a criterion [37], and the strongest association within each LD block was kept. After the clumping of SNPs, 20 QTLs for SDS resistance were identified and peak SNPs (strongest associations) are listed in Table 2. The peak SNPs at the identified loci explained approximately $54.5 \%$ of the phenotypic variance on average (ranging from $35.7 \%$ to $75.4 \%$ for different disease assessment criteria, Figure 4). A major QTL on chromosome 18 was found in both association panels (Figure 3).

\section{QTL confirmation and candidate genes}

We compared the positions of the significant SNPs identified in this study with the positions of the QTLs reported in bi-parental mapping studies and found considerable overlap between these SNPs and the reported genes or QTLs for SDS resistance. Of the 20 loci we detected in the two association panels, seven overlapped with previously identified QTLs (Table 2).

Notably, one of the overlaps is the QTL Rfs $2 / R h g 1$ on chromosome 18. This locus consistently contributes more effective coinheritance of resistance to SDS and reduces infestation by SCN [10]. Previous fine map development did not resolve Rfs 2 from Rhg1, suggesting that the underlying genes were either very closely linked or pleiotropic [11]. In this study, we did detect a cluster of associations spanning a physical region of $1.2 \mathrm{Mb}$ (1.2$2.4 \mathrm{Mb}$ ) around three $R h g 1$ genes that were found to contribute to SCN resistance (Figure 5a, Additional file 9, 10, 11, 12) [51]. The cluster of associations also explained a major part of phenotypic variation of SDS resistance in both panels (Additional file 9, 10, 11, 12). If the three $R h g 1$ genes were pleiotropic, the peak SNP for SDS resistance should be located either within or in the same LD block with the three Rhg1 genes. However, the peak SNP (GM18-1709751) was not only located outside of, but also belonged to a different LD block than the three Rhg1 genes (Additional file 15). One possible explanation for this is that SDS resistance mediated by Rhg1 is also conferred by copy number variation $(\mathrm{CNV})$ that increases the expression of a set of dissimilar genes. Alternatively, there exist other gene/s mediating SDS resistance that are closely linked with 

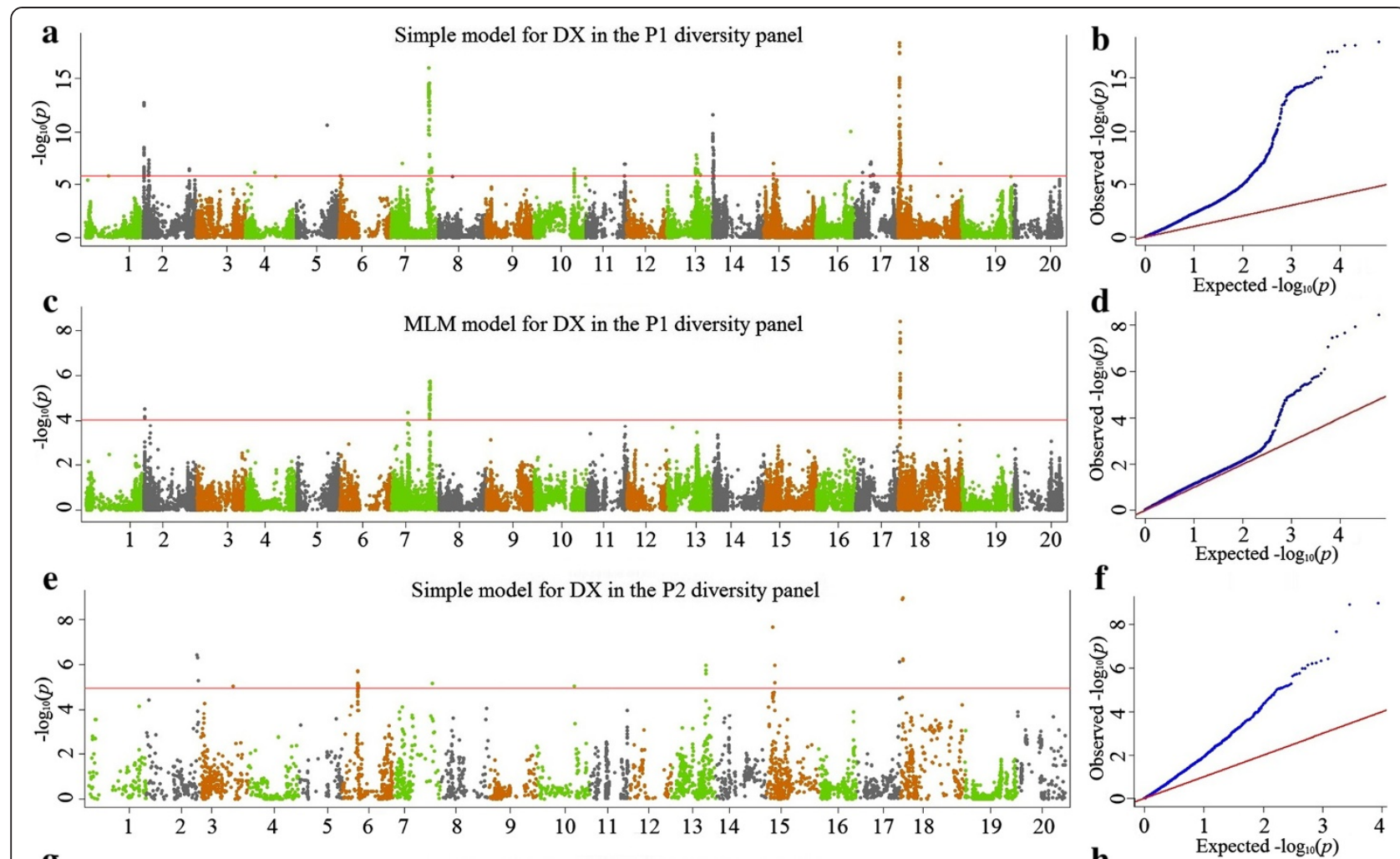

g

MLM model for DX in the P2 diversity panel
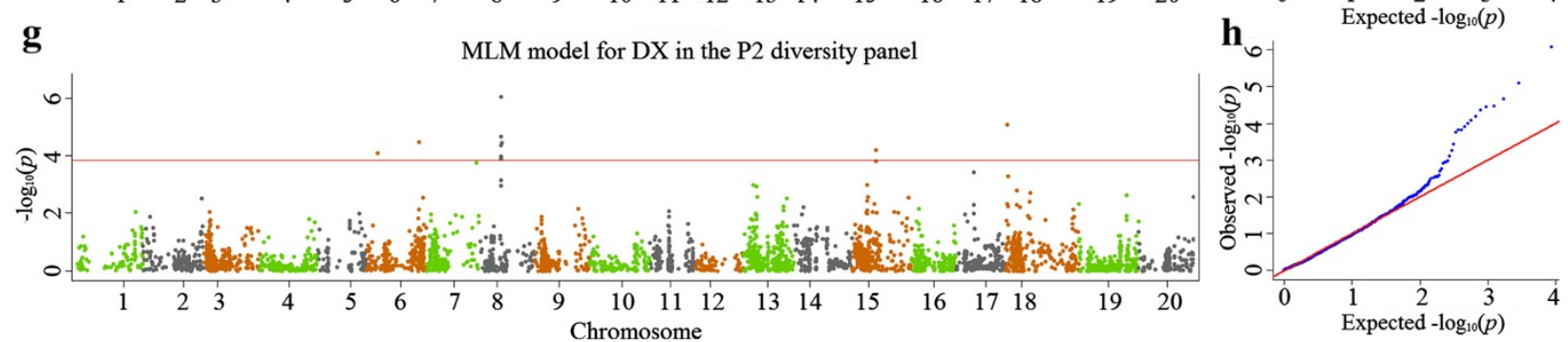

Figure 3 Genome-wide association study of SDS in the two association panels. (a) Manhattan plots of the simple model for DX in the association panel P1. The - $\log 10$ P-values from a genome-wide scan are plotted against the position on each of the 20 chromosomes. The horizontal red line indicates the genome-wide significance threshold (FDR $<0.05)$. (b) Quantile-quantile (QQ) plot of simple model for DX in the association panel P1. (c) Manhattan plots of MLM for DX in association panel P1, as in a. (d) Quantile-quantile plot of MLM for DX in association panel P1. (e) Manhattan plots of the simple model for DX in association panel P2, as in a. (f) Quantile-quantile plot of simple model for DX in the association panel P2.

(g) Manhattan plots of MLM for DX in association panel P2, as in a. (h) Quantile-quantile plot of MLM for DX in association panel P2.

Rhg1. In fact, we found that the peak SNP (GM181709751) was located at approximately $2.2 \mathrm{~kb}$ upstream of GmRLK18-1, a gene that encodes a receptor-like kinase, and its resistance allele is sufficient to confer nearly complete resistance to both root and leaf symptoms of SDS [11]. Moreover, there was another significant SNP (GM18-1712832 with $P$-value of $1.2 \times 10^{-8}$ for DX) located within an exon of GmRLK18-1. These results support previous studies with regard to the key role of GmRLK18-1. However, we cannot exclude the possibility that structural variation in the form of $\mathrm{CNV}$ may have functional importance and thus contribute to SDS resistance that is not captured by our SNPs.

We searched for additional independently associated SNP variants near the $R f s 2 / R h g 1$ locus by conditioning on the peak SNP (GM18-1709751) at the $R f s 2 / R h g 1$ loci. At $519 \mathrm{~kb}$ downstream of $R f s 2 / R h g 1$, we found an independently associated SNP variant, Gm18-2228646 with $P$-value of $6.5 \times 10^{-5}$ in the conditional model, as measured by DS. This SNP is located at 44 bp downstream of a gene encoding an aquaporin transporter (Figure 5b). Therefore, our results provide evidence for the presence of a regulating gene other than GmRLK18-1 that is associated with SDS resistance on chromosome 18.

Besides the Rfs2/Rhg1 region, we refined the mapping location with other significant SNPs within or adjacent to previously reported QTLs (Table 2). Notably, we repeatedly detected a cluster of associations, measured by DS, DI and DX, spanning a physical region of $0.7 \mathrm{Mb}$ (36.5 to $37.2 \mathrm{Mb}$ ) near the $R z d$ locus (resistance to 
Table 2 A subset of SNPs significantly associated with SDS resistance and the adjacent candidate genes

\begin{tabular}{|c|c|c|c|c|c|c|c|}
\hline Trait & SNP & $\mathrm{Chr}^{\mathrm{a}}$ & Position $^{\text {b }}$ & $P$ & $R^{2}(\%)$ & $\mathrm{QTL}^{\mathrm{c}}$ & Candidate genes $^{\mathbf{e}}$ \\
\hline $\mathrm{DX}(\mathrm{P} 1)$ & Gm02-707483 & 2 & 707483 & $3.07 \times 10^{-5}$ & 5.6 & $N^{d}$ & PPR repeat (common disease resistance genes, [38]) \\
\hline $\mathrm{DI}(\mathrm{P} 2)$ & ss244884978 & 2 & 49773810 & $3.60 \times 10^{-4}$ & 6.4 & [39] & Cellulose synthase (disease resistance genes, [40]) \\
\hline $\mathrm{DX}(\mathrm{P} 2)$ & ss 245842048 & 6 & 8979504 & $8.15 \times 10^{-5}$ & 7.7 & N & Phosphatidylinositol kinase (immune responses, [41]) \\
\hline $\mathrm{DX}(\mathrm{P} 2)$ & ss 246038868 & 6 & 43945601 & $3.37 \times 10^{-5}$ & 5.7 & {$[42]$} & LRR gene (pathogen recognition, [43]) \\
\hline DX(P1) & Gm07-15654480 & 7 & 15654480 & $4.36 \times 10^{-5}$ & 5.5 & N & Oxysterol binding protein (upregulated in defense response, [44]) \\
\hline $\mathrm{DS}(\mathrm{P} 1)$ & Gm07-36959086 & 7 & 36959086 & $8.86 \times 10^{-6}$ & 6.5 & N & Ubiquitin-like protein (upregulated in defense response, [45]) \\
\hline $\mathrm{DX}(\mathrm{P} 2)$ & ss 246580442 & 8 & 18469361 & $8.85 \times 10^{-7}$ & 10.9 & N & Zinc finger (disease resistance genes, [46]) \\
\hline $\mathrm{DX}(\mathrm{P} 2)$ & ss246585278 & 8 & 18840490 & $3.55 \times 10^{-5}$ & 8.1 & N & F-box (defense response, [47]) \\
\hline $\mathrm{DI}(\mathrm{P} 1)$ & Gm09-43648118 & 9 & 43648118 & $6.90 \times 10^{-5}$ & 11.6 & N & Phosphopantetheine (disease response, [48]) \\
\hline $\mathrm{DI}(\mathrm{P} 1)$ & Gm11-37426559 & 11 & 37426559 & $2.23 \times 10^{-5}$ & 5.6 & N & Amino acid transporter (disease resistance genes, [49]) \\
\hline $\mathrm{DI}(\mathrm{P} 1)$ & Gm13-4584015 & 13 & 4584015 & $3.50 \times 10^{-6}$ & 7.2 & {$[50]$} & LRR gene (pathogen recognition, [43]) \\
\hline $\mathrm{DX}(\mathrm{P} 2)$ & ss248117124 & 13 & 33655223 & $86 \times 10^{-4}$ & 5.7 & N & Serine/threonine protein kinase (disease defense response, [51]) \\
\hline $\mathrm{DI}(\mathrm{P} 1)$ & Gm14-4636247 & 14 & 4636247 & $6.53 \times 10^{-5}$ & 5.3 & N & Ascorbate oxidase gene (upregulated in defense response, [52]) \\
\hline $\mathrm{DI}(\mathrm{P} 2)$ & ss 248566590 & 15 & 5978279 & $7.98 \times 10^{-4}$ & 5.8 & N & Molecular chaperone (plant defense response, [53]) \\
\hline $\mathrm{DX}(\mathrm{P} 1)$ & ss248698930 & 15 & 20239752 & $6.33 \times 10^{-5}$ & 7.7 & N & Serine/threonine protein kinase (disease defense response, [51]) \\
\hline $\mathrm{DX}(\mathrm{P} 2)$ & ss249511029 & 18 & 1611921 & $8.04 \times 10^{-6}$ & 9.3 & {$[42]$} & Hypoxia induced protein (disease defense signaling, [54]) \\
\hline $\mathrm{DX}(\mathrm{P} 1)$ & Gm18-1709751 & 18 & 1709751 & $3.79 \times 10^{-9}$ & 10.6 & {$[42]$} & Receptor like kinase (disease resistance genes, [11]) \\
\hline $\mathrm{Dl}(\mathrm{P} 2)$ & ss249517154 & 18 & 2113196 & $4.04 \times 10^{-5}$ & 8.3 & {$[42]$} & unknown \\
\hline $\mathrm{DI}(\mathrm{P} 2)$ & ss 249520656 & 18 & 2434513 & $6.9 \times 10^{-6}$ & 9.5 & {$[55]$} & Glycosyltransferase (disease resistance genes, [56]) \\
\hline $\mathrm{DI}(\mathrm{P} 1)$ & Gm19-34890716 & 19 & 34890716 & $2.16 \times 10^{-5}$ & 5.8 & N & Cupins superfamily protein \\
\hline
\end{tabular}

${ }^{a}$ Chromosome; ${ }^{b}$ Position in base pairs for the peak SNP according to soybean reference sequence of Williams $82 ;{ }^{~}$ The candidate gene located in one of the QTL intervals as reported previously and corresponding literature listed in the brackets; ${ }^{d} \mathrm{~N}$ stands for candidates not located in any known QTL intervals; ${ }^{\mathrm{e}} \mathrm{A}$ plausible biological candidate gene in the locus or the nearest annotated gene (Glycine max Wm82.a1.v1) to the peak SNP.

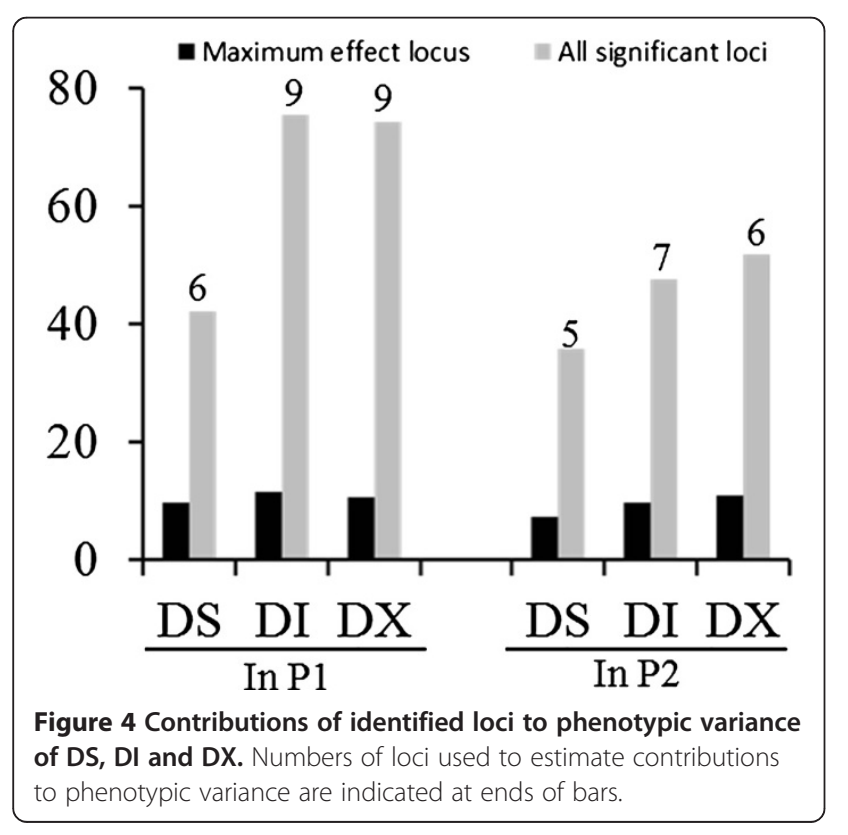

zygote death) on chromosome 7 in panel P1. The Rzd locus contributed to SCN resistance and was strictly coinherited in phase with $R f s 2 / R h g 1$ in an earlier study [57]. However, not all of the QTLs detected in previous bi-parental populations were detected in our association panels. The reason for failure to detect them may be that root infection severity is not included in our disease assessment criteria, so those QTLs associated with resistance to root infection cannot be identified. Alternatively, some QTLs may segregate at low frequency or not at all in our association panels, or the SNP coverage in this study is still insufficient to capture all of the haplotypes present in the diverse soybean varieties. On the other hand, we found 13 novel QTLs. Compared with the 7 loci within intervals of known QTLs, the 13 new loci are slightly weaker in terms of average P-value $\left(6.47 \times 10^{-5}\right.$ vs $\left.7.54 \times 10^{-4}\right)$ and explained phenotypic variance $(8.14 \%$ vs $7.06 \%$ ). However, some of them explain as much as, or even more phenotypic variance than that of known QTLs (Table 2). We checked whether these new QTLs were near loci for determinacy, maturity date, leaf, stem or root morphology and found that one new QTL at 33.6 $\mathrm{Mb}$ on chromosome 13 was within the interval containing QTLs for plant height and stem strength. 


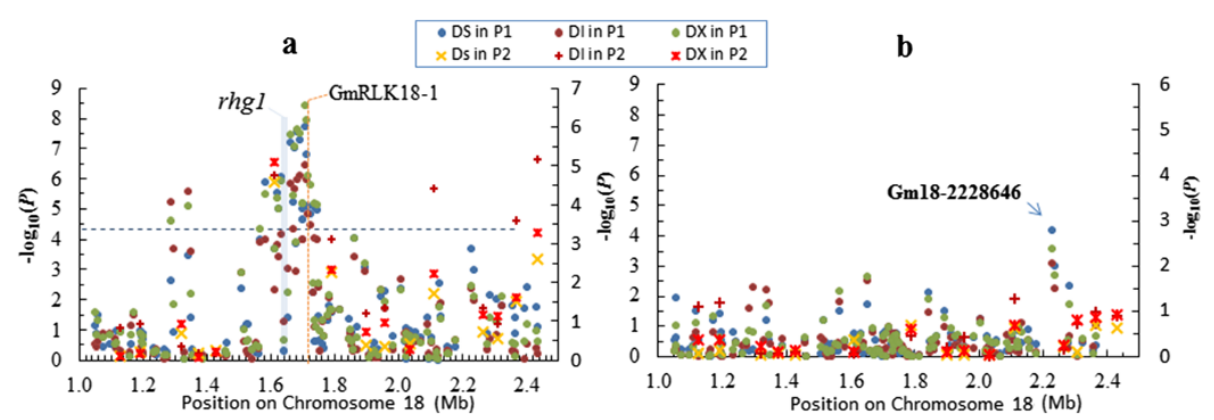

Figure 5 Regional plots showing association mapping results for SNPs located around Rfs2/Rhg1 on chromosome 18. Before (a) and after (b) controlling for the effects of peak SNP, negative log10-transformed $P$-values from the MLM are plotted on the left vertical axis for panel P1; Negative log10-transformed $P$-values from the MLM are plotted on the right vertical axis for association panel P2. Blue horizontal dashed lines indicate the genome-wide significance threshold in P1 association panel. Previously identified genes controlling the SDS resistance are labeled.

Another new QTL at $34.8 \mathrm{Mb}$ on chromosome 19 was located approximately $2 \mathrm{Mb}$ downstream of a locus related with flowering time and leaf morphology. No new QTLs were found near loci related to determinacy, maturity date, or root morphology. To further validate these new loci, we developed five recombinant inbred line (RIL) populations and are currently conducting a confirmation study. To date, we have conducted conventional QTL mapping in three of the five RIL populations. Five out of the 13 novel QTLs have been validated in the three RIL populations (data not shown). Undoubtedly, the major loci identified in this study can be used to improve soybean for SDS resistance.

When we checked candidate genes containing or immediately adjacent to the significant SNPs, we found that diverse types of genes are probably involved in natural variation for soybean SDS resistance (Table 2). For instance, we identified one pentatricopeptide repeat (PPR) gene, which has certain features in common with disease resistance genes ( $\mathrm{R}$ genes) [38]. We also identified two genes encoding leucine-rich repeat (LRR) domains, which are important in plant responses to a variety of external stimuli including pathogens (Table 2 and ref. 44). A gene with similarity to ubiquitin-like protein, which is required for host and nonhost disease resistance in plants [45], was also identified. Several other SNPs were within or adjacent to sequences annotated as homologous to genes previously shown to be involved in plant disease resistance (Table 2). Follow-up studies will focus on validating effects of these genes, uncovering the molecular mechanisms of complex SDS resistance in soybean and integrating this knowledge to dissect mechanisms underlying quantitative resistance to soil-borne pathogens.

\section{Conclusions}

In this study, GWA mapping with correction for population structure and cryptic relatedness identified multiple novel loci and refined the map locations of known loci related to SDS resistance in soybean. This information not only demonstrates that GWA mapping can be used as a powerful tool for dissecting disease resistance mechanisms in soybean, but also provides valuable markers for developing soybean cultivars with durable resistance against SDS. Moreover, the candidate genes containing these SNP loci represent promising targets for further efforts to pinpoint causal variants and to clarify how the implicated genes affect SDS resistance in soybean.

\section{Availability of supporting data}

The data sets supporting the results of this article are included within this article and its additional files.

\section{Additional files}

Additional file 1: Soybean germplasm accessions analyzed in this study. Information is given in this file for each accession, including accession name, origin, maturity group and subpopulation ancestry based Neighbor-joining trees.

Additional file 2: SNPs Distribution of each chromosome on SoySNP 50 k (a) and SoySNP 6 k (b)BeadChip used in genotyping for panel P1 and P2, respectively. This figure is a color index showing the SNP distribution and density of 20 chromosomes on SoySNP $50 \mathrm{k}$ (a) and SoySNP 6 k (b) BeadChip.

Additional file 3: Scale used for phenotyping sudden death syndrome disease severity (DS). Disease incidence (DI) is the percentage of plants in the plot showing leaf symptoms. Disease index $(\mathrm{DX})=(\mathrm{DI} \times \mathrm{DS}) / 9$.

Additional file 4: Kinship value between individual accessions among panels P1 (a) and P2 (b). Individuals are ordered according to their order listed in Additional file 1. Pairwise kinship values are shown as color-index heat map.

Additional file 5: The distributions of minor allele frequencies in P1 (a) and P2 (b) association panels. Two histograms, a for panel P1 and $b$ for panel $\mathrm{P} 2$, showing the distributions of minor allele frequencies in two association panels.

Additional file 6: Phenotypic variation, heritability and correlation analysis in the two association panels. Descriptive statistics information, including mean, range, standard deviation, source of variation and correlation coefficient for DS, DI and DX. 
Additional file 7: Distribution of accessions in each subgroup based on genetic distance in panel P1 and P2. Two-way classification of all accessions, with SNP-data-based clustering (NJ tree) at the top and the maturity-group-based grouping clusters at the left.

Additional file 8: The population differentiation statistics $\left(F_{\mathrm{ST}}\right)$ among subpopulation in panel P1 and P2. Pairwise population differentiation index (Fst) as well as corresponding significant levels are list in this table.

Additional file 9: Associations (FDR $<0.05$ ) identified by GWA mapping for DS in association panel P1. Information of significantly associated SNPs, including name, physical position and phenotypic variation explained by the SNP, is reported in this table.

Additional file 10: Associations (FDR < 0.05 ) identified by GWAS for DI in association panel P1. Information of significantly associated SNPS, including name, physical position and phenotypic variation explained by the SNP, is reported in this table.

Additional file 11: Associations (FDR < 0.05) identified by GWAS for DX in association panel P1. Information of significantly associated SNPs, including name, physical position and phenotypic variation explained by the SNP, is reported in this table.

Additional file 12: Associations (FDR $<0.05$ ) identified by GWAS in association panel P2. Information of significantly associated SNPS, including name, physical position and phenotypic variation explained by the SNP, are reported in this table.

Additional file 13: Genome-wide association study of DS in the two association panels. (a) Manhattan plots of the simple model for DS in association panel P1. The $-\log 10 P$ values from a genome-wide scan are plotted against the position on each of the 20 chromosomes. The horizontal red line indicates the genome-wide significance threshold (FDR < 0.05). (b) Quantile-quantile plot of simple model for DS in the association panel P1. (c) Manhattan plots of MLM for DX in association panel P2, as in a. (d) Quantile-quantile plot of MLM for DS in the panel P1. (e) Manhattan plots of the simple model for DS in association panel P2, as in a. (f) Quantile-quantile plot of simple model for DS in the panel P2. (g) Manhattan plots of MLM for DS in the panel P1, as in a. (h) Quantile-quantile plot of MLM for DS in association panel P2.

Additional file 14: Genome-wide association study of DI in the two association panels. (a) Manhattan plots of the simple model for DI in association panel P1. The $-\log 10 P$ values from a genome-wide scan are plotted against the position on each of the 20 chromosomes. The horizontal red line indicates the genome-wide significance threshold (FDR $<0.05)$. (b) Quantile-quantile plot of simple model for DI in the association panel P1. (c) Manhattan plots of MLM for DX in association panel P1, as in a. (d) Quantile-quantile plot of MLM for DI in association panel P1. (e) Manhattan plots of the simple model for DI in association panel P2, as in a. (f) Quantile-quantile plot of simple model for DI in the association panel P2. (g) Manhattan plots of MLM for DI in association panel P2, as in a. (h) Quantile-quantile plot of MLM for DI in association panel P2.

Additional file 15: Regional plots showing association mapping results for SNPs located around Rfs2/Rhg1 on chromosome 18. Negative log10-transformed $P$-values from the MLM are plotted on the left vertical axis for association panel P1; Negative log10-transformed $P$-values from the MLM are plotted on the right vertical axis for association panel P2. Blue horizontal dashed lines indicate the genome-wide significance threshold in association panel P1. Previously identified genes controlling the traits are labeled.

\section{Abbreviations}

SDS: Sudden death syndrome; SCN: Soybean cyst nematode; DI: Disease incidence; DS: Disease severity; DX: Disease index; GWA: Genome-wide association; SNPs: Single nucleotide polymorphisms; LD: Linkage disequilibrium; QTL: Quantitative trait locus; SSR: Simple sequence repeat; GLM: General linear model; MLM: Mixed linear model.

\section{Competing interests}

The authors declare that they have no competing interests.

\section{Authors' contributions}

DW designed and supervised the research; ZW performed the SNP genotyping, analyzed the data and wrote the manuscript; RT, JY, CB, WD, SZ, MC, CS conducted the field investigation; QS and PBC developed SNP chips; All authors read and approved the manuscript.

\section{Acknowledgments}

We thank all the participants in the 2011 and 2012 Soybean Sudden Death Syndrome Regional Tests who provided the SDS resistance data and/or the seeds of the tested soybean genotypes. The participants were J. Bond, R. Bowen, P. Chen, S. Cianzio, B. Diers, A. Dorrance, K. Gallo, S. Kantartzi, D. Kyle, D. Malvick, J.H. Orf, H. Ramasubramaniam, and T. Welacky. We also thank C. and A. Druskovich for use of their field and J. Boyse, N. Boyse, R. Laurenz, B. Serven and J. Jacobs for technical assistance. We appreciate Dr. Linda Kull for her coordination of the NCSRP SDS Research Alliance which included this study. We thank Dr. Frank Dennis and Paul Collins for editing. Funding was provided by the North Central Soybean Research Program and Michigan Soybean Promotion Committee.

\section{Author details}

'Department of Plant, Soil and Microbial Sciences, Michigan State University, 1066 Bogue St., Rm. A384-E, East Lansing, Ml 48824, USA. ${ }^{2}$ Agronomy Research Center, Southern Illinois University Carbondale, Carbondale, Illinois 62903-7002, USA. ${ }^{3}$ USDA, Agricultural Research Service, Soybean Genomics and Improvement Laboratory, Beltsville, Maryland 20705, USA.

Received: 8 May 2014 Accepted: 18 August 2014

Published: 23 September 2014

\section{References}

1. Roy KW, Rupe JCD, Hershman DE, Abney TS: Sudden death syndrome of soybean. Plant Dis 1997, 81:1100-1111.

2. Wrather JA, Shannon G, Balardin R, Carregal L, Escobar R, Gupta GK, Ma Z, Morel W, Ploper D, Tenuta A: Effect of diseases on soybean yield in the top eight producing countries in 2006. Plant Health Progress 2010, doi:10.1094/PHP-2010- 0125-01-RS.

3. Jin $\mathrm{H}$, Hartman GL, Nickell CD, Widholm JM: Characterization and purification of a phytotoxin produced by Fusarium solani, the causal agent of soybean sudden death syndrome. Phytopathology 1996, 86:277-282.

4. Rupe JC, Hartman GL: Sudden death syndrome. In Compendium of Soybean Diseases. Edited by Hartman GL, Sinclair JB, Rupe JC. St. Paul: APS Press; 1999:37-39.

5. Wrather JA, Koenning SR: Estimates of disease effects on soybean yields in the United States 2003 to 2005. J Nematology 2006, 38:173-180.

6. Wrather JA, Koenning SR: Effects of diseases on soybean yields in the United States 1996 to 2007. Plant Health Progr 2009, doi:10.1094/PHP-20090401-01-RS.

7. de Farias Neto AL, Hartman GL, Pedersen WL, Li S, Bollero GA, Diers BW: Mapping and confirmation of a new sudden death syndrome resistance QTL on linkage group D2 from the soybean genotypes PI567374 and 'Ripley'. Mol Breed 2007, 20:53-62.

8. Hartman GL, Huang YH, Nelson RL, Noel GR: Germplasm evaluation of Glycine max for resistance to Fusarium solani, the causal organism of sudden death syndrome. Plant Dis 1997, 81:515-551.

9. Njiti VN, Johnson JE, Torto TA, Gray LE, Lightfoot DA: Inoculum rate influences selection for field resistance to sudden death syndrome in the greenhouse. Crop Sci 2001, 41:1726-1733.

10. Luckew AS, Leandro LF, Bhattacharyya MK, Nordman DJ, Lightfoot DA Cianzio SR: Usefulness of 10 genomic regions in soybean associated with sudden death syndrome resistance. Theor Appl Genet 2013, 126:2391-2403

11. Srour A, Afzal AJ, Blahut-Beatty L, Hemmati N, Simmonds DH, Li W, Liu M, Town CD, Sharma H, Arelli P, Lightfoot DA: The receptor like kinase at Rhg1-a/Rfs2 caused pleiotropic resistance to sudden death syndrome and soybean cyst nematode as a transgene by altering signaling responses. BMC Genomics 2012, 13:368. doi:10.1186/1471-2164-13-368.

12. Zhu CS, Gore M, Buckler ES, Yu JM: Status and prospects of association mapping in Plants. Plant Gen 2008, 1:5-20.

13. Atwell $S$, Huang YS, Vilhjálmsson BJ, Willems G, Horton M, Li Y, Meng D, Platt A, Tarone AM, Hu TT, Jiang R, Muliyati NW, Zhang X, Amer MA, 
Baxter I, Brachi B, Chory J, Dean C, Debieu M, de Meaux J, Ecker JR, Faure N, Kniskern JM, Jones JD, Michael T, Nemri A, Roux F, Salt DE, Tang C, Todesco M, et al: Genome-wide association study of 107 phenotypes in Arabidopsis thaliana inbred lines. Nature 2010, 465:627-631.

14. Huang X, Wei X, Sang T, Zhao Q, Feng Q, Zhao Y, Li C, Zhu C, Lu T, Zhang Z, Li M, Fan D, Guo Y, Wang A, Wang L, Deng L, Li W, Lu Y, Weng Q, Liu K, Huang T, Zhou T, Jing Y, Li W, Lin Z, Buckler ES, Qian Q, Zhang QF, Li J, Han B: Genomewide association studies of 14 agronomic traits in rice landraces. Nat Genet 2010, 42(11):961-967.

15. Kump KL, Bradbury PJ, Wisser RJ, Buckler ES, Belcher AR, Oropeza-Rosas MA, Zwonitzer JC, Kresovich S, McMullen MD, Ware D, Balint-Kurti PJ, Holland JB: Genome-wide association study of quantitative resistance to southern leaf blight in the maize nested association mapping population. Nat Genet 2011, 43:163-168.

16. Tian F, Bradbury PJ, Brown PJ, Hung H, Sun Q, Flint-Garcia S, Rocheford TR, McMullen MD, Holland JB, Buckler ES: Genome-wide association study of leaf architecture in the maize nested association mapping population. Nat Genet 2010, 43:159-162.

17. Hao D, Cheng H, Yin Z, Cui S, Zhang D, Wang H, Yu D: Identification of single nucleotide polymorphisms and haplotypes associated with yield and yield components in soybean (Glycine max) landraces across multiple environments. Theor App/ Genet 2012, 124:447-458.

18. Wang J, McClean PE, Lee R, Goos RJ, Helms T: Association mapping of iron deficiency chlorosis loci in soybean (Glycine max L. Merr.) advanced breeding lines. Theor App/ Genet 2008, 116:777-787.

19. Schmutz J, Cannon SB, Schlueter J, Ma J, Mitros T, Nelson W, Hyten DL, Song Q, Thelen JJ, Cheng J, Xu D, Hellsten U, May GD, Yu Y, Sakurai T, Umezawa T, Bhattacharyya MK, Sandhu D, Valliyodan B, Lindquist E, Peto M, Grant D, Shu S, Goodstein D, Barry K, Futrell-Griggs M, Abernathy B, Du J, Tian Z, Zhu L, et al: Genome sequence of the palaeopolyploid soybean. Nature 2010, 463:178-183.

20. Song Q, Hyten DL, Jia G, Quigley CV, Fickus EW, Nelson RL, Cregan PB: Development and evaluation of SoySNP50K, a high-density genotyping array for soybean. PLOS ONE 2013, 8(1):e54985. doi:10.1371/journal.pone.0054985.

21. Muller DS, Hartman GL, Nelson RL, Pedersen WL: Evaluation of Glycine max germplasm for resistance to Fusarium solani f. sp. glycines. Plant Disease 2002, 86:741-746.

22. Gelin JR, Arelli PR, Rojas-Cifuentes PR: Using independent culling to screen plant introductions for combined resistance to soybean cyst nematode and sudden death syndrome. Crop Sci 2006, 46:2081-2083.

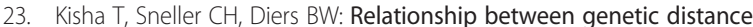
among parents and genetic variance in populations of soybean. Crop Sci 1997, 37:1317-1325.

24. Akond M, Liu S: A SNP-based genetic linkage map of soybean using the SoySNP6K Illumina Infinium BeadChip genotyping array. J Plant Genom Sci 2013, 1(3):80-89.

25. Yan J, Yang X, Shah T, Sánchez-Villeda H, Li J, Warburton M, Zhou Y, Crouch JH, Xu Y: High-throughput SNP genotyping with the Golden Gate assay in maize. Mol Breed 2010, 25:441-451.

26. Fehr WR, Caviness CE, Burmood DT, Pennington JS: Stage of development descriptions for soybeans, Glycine max (L.)Merrill. Crop Sci 1971, 11:929-931.

27. Nyquist WE: Estimation of heritability and prediction of selection response in plant populations. Crit Rev Plant Sci 1991, 10:235-322.

28. Liu K, Muse SV: PowerMarker: integrated analysis environment for genetic marker data. Bioinformatics 2005, 21:2128-2129.

29. Price AL, Patterson NJ, Plenge RM, Weinblatt ME, Shadick NA, Reich D: Principal components analysis corrects for stratification in genome-wide association studies. Nat Genet 2006, 38:904-909.

30. Bradbury PJ, Zhang Z, Kroon DE, Casstevens TM, Ramdoss Y, Buckler ES: TASSEL: software for association mapping of complex traits in diverse samples. Bioinformatics 2007, 23:2633-2635.

31. Yu J, Pressoir G, Briggs WH, Vroh Bi I, Yamasaki M, Doebley JF, McMullen MD, Gaut BS, Nielsen DM, Holland JB, Kresovich S, Buckler ES: A unified mixed-model method for association mapping that accounts for multiple levels of relatedness. Nat Genet 2006, 38:203-208.

32. Zhang Z, Ersoz E, Lai CQ, Todhunter RJ, Tiwari HK, Gore MA, Bradbury PJ, Yu J, Arnett DK, Ordovas JM, Buckler ES: Mixed linear model approach adapted for genome-wide association studies. Nat Genet 2010, 42:355-360.
33. Li YH, Li W, Zhang C, Yang L, Chang RZ, Gaut BS, Qiu LJ: Genetic diversity in domesticated soybean (Glycine max) and its wild progenitor (Glycine soja) for simple sequence repeat and single-nucleotide polymorphism loci. New Phytologist 2010, 188:242-253.

34. Hyten DL, Choi IY, Song Q, Shoemaker RC, Nelson RL, Costa JM, Specht JE, Cregan PB: Highly variable patterns of linkage disequilibrium in multiple soybean populations. Genetics 2007, 175:1937-1944.

35. Yan J, Shah T, Warburton ML, Buckler ES, McMullen MD, Crouch J: Genetic characterization and linkage disequilibrium estimation of a global maize collection using SNP markers. PLOS ONE 2009, 4(12):8451. doi:10.1371 /journal. pone.0008451

36. Roberts EH, Qi A, Ellis RH, Summerfield RJ, Lawn RJ, Shanmugasundaram S: Use of field observations to characterize genotypic flowering responses to photoperiod and temperature: A soybean exemplar. Theor App/ Genet 1996, 93:519-533.

37. Gabriel SB, Schaffner SF, Nguyen H, Moore JM, Roy J, Blumenstiel B, Higgins J, DeFelice M, Lochner A, Faggart M, Liu-Cordero SN, Rotimi C, Adeyemo A, Cooper R, Ward R, Lander ES, Daly MJ, Altshuler D: The structure of haplotype blocks in the human genome. Science 2002, 296:2225-2229.

38. Geddy R, Brown GG: Genes encoding pentatricopeptide repeat (PPR) proteins are not conserved in location in plant genomes and may be subject to diversifying selection. BMC Genomics 2007, 8:130. doi:10.1186/ 1471-2164-8-130.

39. Kassem MA, Ramos L, Leandro L, Mbofung G, Hyten DL, Kantartzi SK, Grier RL IV, Njiti VN, Cianzio S, Meksem K: The 'PI 438489B' by 'Hamilton' SNP-based genetic linkage map of soybean [Glycine max (L.) Merr.] identified quantitative trait loci that underlie seedling SDS resistance. J Plant Genome Sci 2012, 1:18-30.

40. Hernández-Blanco C, Feng DX, Hu J, Sánchez-Vallet A, Deslandes L, Llorente F, Berrocal-Lobo M, Keller H, Barlet X, Sánchez-Rodríguez C, Anderson LK, Somerville S, Marco Y, Molina A: Impairment of cellulose synthases required for Arabidopsis secondary cell wall formation enhances disease resistance. Plant Cell 2007, 19:890-903.

41. Yaeno T, Li H, Chaparro-Garcia A Schornack S, Koshiba S, Watanabe S, Kigawa T, Kamoun S, Shirasu K: Phosphatidylinositol monophosphatebinding interface in the oomycete RXLR effector AVR3a is required for its stability in host cells to modulate plant immunity. Proc Natl Acad Sci USA 2011, 108(35):14682-14687.

42. Iqbal MJ, Meksem K, Njiti VN, Kassem MA, Lightfoot DA: Microsatellite markers identify three additional quantitative trait loci for resistance to soybean sudden-death syndrome (SDS) in Essex Forrest RILs. Theor Appl Genet 2001, 102:187-192.

43. Bent AF, Mackey D: Elicitors, effectors, and R genes: The new paradigm and a lifetime supply of questions. Annu Rev Phytopathol 2007, 45:399-436.

44. Avrova AO, Taleb N, Rokka VM, Heilbronn J, Campbell E, Hein I, Gilroy EM, Cardle L, Bradshaw JE, Stewart HE, Fakim YJ, Loake G, Birch PR: Potato oxysterol binding protein and cathepsin $B$ are rapidly up-regulated in independent defence pathways that distinguish $\mathrm{R}$ gene-mediated and field resistances to Phytophthora infestans. Mol Plant Pathol 2004, 5(1):45-56.

45. Peart JR, Lu R, Sadanandom A, Malcuit I, Moffett P, Brice DC, Schauser L, Jaggard DA, Xiao S, Coleman MJ, Dow M, Jones JD, Shirasu K, Baulcombe DC: Ubiquitin ligase-associated protein SGT1 is required for host and nonhost disease resistance in plants. Proc Natl Acad Sci USA 2002, 99(16):10865-10869.

46. Gupta SK, Rai AK, Kanwar SS, Shama TR: Comparative analysis of zinc finger proteins involved in plant disease resistance. PLOS ONE 2012, 7(8):e42578. doi:10.1371/ journal.pone.0042578.

47. Kim HS, Delaney TP: Arabidopsis SON1 is an F-box protein that regulates a novel induced defense response independent of both salicylic acid and systemic acquired resistance. Plant Cell 2002, 14(7):1469-1482.

48. Rock CO, Park HW, Jackowski S: Role of feedback regulation of pantothenate kinase (CoaA) in control of coenzyme A levels in Escherichia coli. J Bacteriol 2003, 185(11):3410-3415.

49. Liu G, Ji Y, Bhuiyan NH, Pilot G, Selvaraj G, Zou J, Wei Y: Amino acid homeostasis modulates salicylic acid-associated redox status and defense responses in Arabidopsis. Plant Cell 2010, 22(11):3845-3863.

50. Kassem MA, Shultz J, Meksem K, Cho Y, Wood AJ, lqbal MJ, Lightfoot DA: An updated Essex by Forrest linkage map and first composite interval map of QTL underlying six soybean traits. Theor Appl Genet 2006, 113:1015-1026. 
51. Cook DE, Lee TG, Guo X, Melito S, Wang K, Bayless AM, Wang J, Hughes TJ, Willis DK, Clemente TE, Diers BW, Jiang J, Hudson ME, Bent AF: Copy number variation of multiple genes at Rhg1 mediates nematode resistance in soybean. Science 2012, 338:1206-1209.

52. Mysore KS, D'Ascenzo MD, He X, Martin GB: Overexpression of the disease resistance gene Pto in tomato induces gene expression changes similar to immune responses in human and fruitfly. Plant Physiol 2003, 132(4):1901-1912

53. Liu Y, Burch-Smith T, Schiff M, Feng S, Dinesh-Kumar SP: Molecular chaperone $\mathrm{Hsp} 90$ associates with resistance protein $\mathrm{N}$ and its signaling proteins SGT1 and Rar1 to modulate an innate immune response in plants. J Biol Chem 2004, 279(3):2101-2108.

54. Steffens B, Sauter M: G proteins as regulators in ethylene-mediated hypoxia signaling. Plant Signal Behav 2010, 5(4):375-378.

55. Kazi S, Shultz J, Afzal J, Johnson J, Njiti VN, Lightfoot DA: Separate loci underlie resistance to root infection and leaf scorch during soybean sudden death syndrome. Theor Appl Genet 2008, 116:967-977.

56. Lorenc-Kukuła K, Zuk M, Kulma A, Czemplik M, Kostyn K, Skala J, Starzycki M, Szopa J: Engineering flax with the GT family 1 Solanum sogarandinum glycosyltransferase SsGT1 confers increased resistance to Fusarium infection. J Agric Food Chem 2009, 57(15):6698-6705.

57. Webb DM, Baltazar BM, Rao-Arelli AP, Schupp J, Clayton K, Keim P, Beavis WD: Genetic mapping of soybean cyst nematode race- 3 resistance loci in the soybean PI 437654. Theor Appl Genet 1995, 91:574-581.

doi:10.1186/1471-2164-15-809

Cite this article as: Wen et al: Genome-wide association mapping of quantitative resistance to sudden death syndrome in soybean. BMC Genomics 2014 15:809.

\section{Submit your next manuscript to BioMed Central and take full advantage of:}

- Convenient online submission

- Thorough peer review

- No space constraints or color figure charges

- Immediate publication on acceptance

- Inclusion in PubMed, CAS, Scopus and Google Scholar

- Research which is freely available for redistribution 